\title{
Gender Differences in Stigma and HIV-Related Quality of Life in People Living with HIV
}

Brittney M. Woods ${ }^{1}$, Erin M. Fekete, Ph.D. ${ }^{1}$, Stacey L. Williams, Ph.D. ${ }^{2}$ Matthew D. Skinta, Ph.D. ${ }^{3}$, Nicole M. Taylor, Ph.D. ${ }^{1}$ Michael Chatterton, M. A. ${ }^{1}$, Megan M. White, M. A. ${ }^{1}$

${ }^{1}$ University of Indianapolis, ${ }^{2}$ East Tennessee State University, ${ }^{3}$ Private Practice, San Francisco, CA First Author Contact Information: woodsb@uindy.edu

Principal Investigator Contact Information: feketee@uindy.edu

\section{ABSTRACT}

We hypothesized that HIV-related stigma would be related to poorer HIV-related quality of life (HIV-QOL) in people living with HIV (PLWH), and that this relationship would be stronger in women living with HIV (WLWH) than in men ling wh stigma, and HIV-OOL. Results suggest that higher levels of HIV-stigma were associated with poorer HIV-QOL, and that in some cases, this relationship was stronger for WLWH than for MLWH. It is possible that WLWH have unique HIV-related experiences affecting their quality of life that are not shared by MLWH.

\section{INTRODUCTION}

HIV-related stigma is the felt or enacted negative attitudes or maltreatment that an individual

experiences as a consequence of their HIV-status.

- Associated with poorer psychological and

Associated with poorer psychological
physical well-being, poorer medication

adherence, and poorer health related quality of life.

HIV-related quality of life (HIV-QOL) impacts the health and disease management of a person living with HIV.

Medication Adherence, Disclosure Worries, Social Support, Coping, Comorbid Disorders

Compared to MLWH, WLWH may experience more

HIV-stigma and have poorer HIV-QOL.

Fewer economic and social resources in coping

with their illness.

Different health worries than MLWH, including

passing the infection to offspring.

- Caregiving and other family responsibilities that may take precedence over self-care.

\section{HYPOTHESES}

We predicted that higher levels of HIV-related stigma would be associated with lower HIV-related quality of fe in both WLWH and MLWH.

We also expected that the relationship between stigma and lower HIV-related quality of life would be stronger for WLWH than for MLWH.

\begin{tabular}{|c|c|}
\hline \multicolumn{2}{|c|}{ PARTICIPANTS ( $\mathbf{N}=105)$} \\
\hline Gender & Male $=70$, Female $=35$ \\
\hline Age & $M=42.7$ years old \\
\hline Years with HIV & $M=11.2$ years \\
\hline Education & $\begin{array}{l}\text { Did Not Graduate High School }= \\
5.7 \% \text {, High School Graduate }= \\
74.3 \% \text {, College Graduate }=20.0 \%\end{array}$ \\
\hline $\begin{array}{l}\text { Median Household } \\
\text { Income }\end{array}$ & $\$ 10,000$ to $\$ 14,999$ annually \\
\hline Race & $\begin{array}{l}\text { 49\% Black, 36\% White, 13\% } \\
\text { Hispanic, } 2 \% \text { Biracial }\end{array}$ \\
\hline
\end{tabular}

PROCEDURE AND MEASURES

- Participants completed an online survey, including: - Demographics

- The HIV-Stigma Scale

HIV-AIDS Targeted Quality of Life Instrument (HAT-QOL)

Upon completion, \$20.00 gift cards were mailed to participants.

\begin{tabular}{|l|c|c|c|c|c|}
\hline \multicolumn{1}{|c|}{ Measure } & Mean & SD & $\begin{array}{c}\text { Actual } \\
\text { Range }\end{array}$ & $\begin{array}{c}\text { Potential } \\
\text { Range }\end{array}$ & $\boldsymbol{\alpha}$ \\
\hline HIV-Stigma & 97.52 & 25.5 & $43-153$ & $41-164$ & 0.96 \\
\hline $\begin{array}{l}\text { Life } \\
\text { Satisfaction }\end{array}$ & 54.35 & 32.2 & $0-100$ & $0-100$ & 0.94 \\
\hline $\begin{array}{l}\text { Overall } \\
\text { Functioning }\end{array}$ & 65.53 & 25.0 & $0-100$ & $0-100$ & 0.86 \\
\hline Health Worries & 70.60 & 28.4 & $0-100$ & $0-100$ & 0.88 \\
\hline $\begin{array}{l}\text { Financial } \\
\text { Worries }\end{array}$ & 51.59 & 35.3 & $0-100$ & $0-100$ & 0.92 \\
\hline $\begin{array}{l}\text { Medication } \\
\text { Worries }\end{array}$ & 73.16 & 28.5 & $0-100$ & $0-100$ & 0.90 \\
\hline HIV-Mastery & 61.43 & 36.3 & $0-100$ & $0-100$ & 0.89 \\
\hline $\begin{array}{l}\text { Disclosure } \\
\text { Worries }\end{array}$ & 63.76 & 29.5 & $0-100$ & $0-100$ & 0.86 \\
\hline $\begin{array}{l}\text { Provider } \\
\text { Treatment }\end{array}$ & 72.26 & 31.8 & $0-100$ & $0-100$ & 0.94 \\
\hline $\begin{array}{l}\text { Sexual } \\
\text { Functioning }\end{array}$ & 64.29 & 34.3 & $0-100$ & $0-100$ & 0.93 \\
\hline
\end{tabular}

\section{ANALYSIS PLAN}

Covariates:

Any demographic, health, or socia

characteristics associated with the dependen

variables.

Hierarchical Linear Regression and Moderated

Regression Analyse

Block 1: Covariates

Block 2: Centered Pred

Block 3: Moderator (gender)

Block 4: Interaction term (stigma $x$ gender)

Significant interactions were decomposed using simple slopes analysis.

\section{MAIN EFFECTS}

Higher levels of HIV-related stigma are associated with several dimensions of poorer HIV-related OOL: - More health worries, financial worries, medication worries, disclosure worries

- Less HIV-mastery

- Poorer sexual functioning

\begin{tabular}{|l|c|c|c|}
\hline HIV-Quality of Life & $\boldsymbol{\beta}$ & $\mathbf{S E}$ & $\boldsymbol{\Delta R}^{\mathbf{2}}$ \\
\hline Life Satisfaction & -.14 & .10 & .02 \\
\hline Overall Function & -.16 & .08 & .02 \\
\hline Health Worries & -.37 & .08 & $.12^{\star \star \star}$ \\
\hline Financial Worries & -.48 & .09 & $.20^{\star \star}$ \\
\hline Medication Worries & -.40 & .09 & $.13^{\star \star}$ \\
\hline HIV-Mastery & -.36 & .10 & $.11^{\star \star \star}$ \\
\hline Disclosure Worries & -.53 & .08 & $.23^{\star \star \star}$ \\
\hline Provider Treatment & .02 & .10 & .00 \\
\hline Sexual Function & -.31 & .09 & $.08^{\star \star \star}$ \\
\hline
\end{tabular}

Note. Higher scores on the HAT-QOL indicate better quality of lite

\section{INTERACTION EFFECTS}

- Significant interaction effects emerged between stigma and gender in explaining health worries $(\beta$ functioning $\left(\beta=-21, S E=10, p<05, \Delta R^{2}=.03\right)$.

HEALTH WORRIES

${ }^{120}$

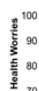

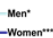

SEXUAL FUNCTIONING

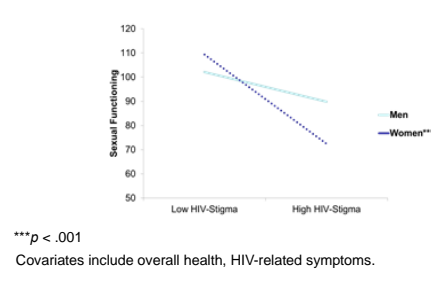

DISCUSSION

The gender disparities in HIV disease progression may be explained, in part, by gender differences

HIV-related stigma and HIV-QOL.

Although HIV-related stigma impacts HIV-QOL in both MLWH and WLWH, HIV-related stign has a stronger impact on some aspects of WLWH'S HIV-QOL

WLWH occupy different social, economic, and work roles than MLWH.

- igher rates of child related stress, more interpersonal violence, fewer social

Psychosocial interventions to reduce HIV-related be tailored to the unique needs of WLWH. 